\title{
Motion in Clifford Space
}

\author{
Magd E. Kahil ${ }^{1,2}$ \\ ${ }^{1}$ Faculty of Engineering, Modern Sciences and Arts University, Giza, Egypt \\ ${ }^{2}$ Egyptian Relativity Group, Cairo, Egypt \\ Email: e.mailmkahil@msa.eun.eg, magdelias.kahil@gmail.com
}

How to cite this paper: Kahil, M.E. (2020) Motion in Clifford Space. Journal of Modern Physics, 11, 1856-1873.

https://doi.org/10.4236/jmp.2020.1111116

Received: October 22, 2020

Accepted: November 17, 2020

Published: November 20, 2020

Copyright (c) 2020 by author(s) and Scientific Research Publishing Inc.

This work is licensed under the Creative Commons Attribution International License (CC BY 4.0).

http://creativecommons.org/licenses/by/4.0/

\section{Abstract}

Clifford algebra as an approach of geometrization of physics plays a vital role in unification of micro-physics and macro-physics, which leads to examine the problem of motion for different objects. Equations of charged and spinning of extended objects are derived. Their corresponding deviation equations as an extension of geodesics and geodesic deviation of vectors in Riemannian geometry have been developed in case of Clifford space.

\section{Keywords}

Cifford Space, Poly-Vectors-Geodesics, Geodesic Deviation, Spinning Objects, Extended Objects

\section{Clifford Space: Aims and Prospects}

Motion of objects is regarded as a mirror to identify the behavior of field equations on manifolds. This may give rise to examine the trajectories of different particles to ensure the existence of any theory and its viability. From this perspective, we ought to study the problem of spinning objects in depth, as it is very close to the reality, rather than examining its simplicity by means of determining the equation of motion of test particles, i.e. the geodesic equation. The spinning object has been studied by many authors long time ago, Mathisson [1] started the idea; Papapetrou amended its content [2] and then it was developed to include charged objects by Dixon [3], which led many of their followers to obtain the corresponding equations of motion of moving objects in different types of geometries [4-9]. Not only these path equations but also their deviation equations play a fundamental role in regulating the stability of objects [10]. This is mandatory in case of examining the perturbation problem of an object orbiting a gravitational field. Yet, such a description may be in need to be revisited thoroughly for sake of unification of physics. Since the problem of unification of all fields of nature is a far fetched goal, it is wise enough to search for different methods and concepts that enable us to achieve this goal one day. 
Accordingly, examining the problem of unification may be found by studying the motion of particles subject to these fields. This may give rise to search for different types of geometries or philosophies to achieve this task. One of proposals is to reconsider a specific geometry based strings, 1 loop, 2-loops, 3-loops, etc. In general it can be described as p-branes rather than points. This shows vectors as candidates of poly-vectors to represent objects in nature as extended ones rather than point-like. Consequently, it has been found that Clifford Algebra is a good candidate to describe these quantities, leading to the concept of Clifford space (C-space) [11]. From this perspective, and relying to examine the problem of motion for those extended objects, Pezzaglia (1997) performed an introductory step toward this task by studying the equations of motion $[11,12]$. Throughout his work, he focussed on the nature of the bases of C-space that are non-orthogonal, which widens the scope to reveal some obscure relations that are already untested. Such an approach has been given an additional role for quantities like the tetrad formalism as a tool for defining skew symmetric quantities like torsion of space-time, and spin connection in non-Riemaniann geometries. This tendency would be able to revise the absolute parallelism approach [13-15], as well as the Poincare gauge theories of gravity $[16,17]$. Moreover, it gives a clear vision to detect the effect of spin of space-time as appeared in skew part of $g_{\mu \nu}$ [12]. Accordingly, the principle geometrization of physics has been applied in an extensive way rather than before in the conventional point-like manifold theories [18] .

Consequently, the concept of a manifold composing points to identify space-time is amended to include, lines, ares, volumes, ... etc. According to this descriptive vision particles may be defined as extended objects, rather than point-like ones [19]. This is performed by means of applying the Clifford algebra, which is regarded as pandimensional continuum or Clifford space (C-space). Due to this type of classification it is worth mentioning that, Castro and Pavsic (2005) revisited Clifford space to perform its extended relativity [18]. This theory has two fundamental parameters: the speed of light $c$ and a length scale which can be set to the Planck length. The poly vector coordinates $x^{\mu}, x^{\mu \nu}, x^{\mu \nu \rho} \ldots$ are now connected with basis poly-vector, bi-vector, tri-vector, $\ldots$ r-vector, the generators $\gamma_{\mu_{1}} \wedge \ldots \wedge \ldots \wedge \gamma_{\mu_{r}}$ of the Clifford algebra, including the Clifford algebra unit element [20].

From this perspective, it can be found that strings and p-branes are expressed in the following way:

For a closed string (1-loop) which is embedded in a target flat background of D-dimensions whose projection is appeared within coordinate-planes in terms of variables $x_{\mu \nu}$. Similarly, a closed membrane (a 2-loop) may be described by anti-symmetric variables $x_{\mu \nu \rho}$ representing the corresponding 3 -volume enclosed by the the 2 -loop [21].

The aim of the present work is to extend the Castro-Pavsic approach of poly-vectors in C-space to derive modified equations of motion for extended objects and spinning ones. The significance of the derivation of these equations is in examining the existence of the notion of mass, charge and spin of an extended object associated with poly-vectors and compare them with the conventional ones as described within the context of vectors.

The paper is organized as follows: Section (2) displays briefly the geometry of C-space and its implications in physics. Section (3) per- 
forms the trajectories of poly-vectors in C-space. Section (4) discusses the relationship between geodesics and geodesic deviations of polyvectors and their link with charged and spinning poly-vectors. Section (5) displays a modified Lagrangian which enables us to determine both charged and spinning poly-vector equations as well as their deviation equations. Section (6) discusses the impact of determining these equations in revisiting the old notations of problem of motion using the conventional methods of a point-like manifold of describing space-time.

\section{C-Space: A Brief Introduction}

Following the geometrization scheme of physics, a new elegant formalism has been performed, which may explore new hidden physics to show an insightful vision for revisiting the old notations of physics. Such a trend is a crystal clear using Clifford Algebra which leads to express many physical quantities in a compact form [22]. Due to the richness of Clifford algebra, scalars, vectors, bi-vectors and r-vectors are expressed in one form called Clifford aggregate or poly-vector. The coordinates of poly-vectors contain, vectors, areas, volumes ... etc expressed as follows The poly-vector $X^{M}$ is defined as follows [23]

$$
X^{M} \equiv X^{\mu_{1} \mu_{2} \mu_{3} \cdots} .
$$

Accordingly, it can be shown that poly-vector coordinates of Cspace are parameterized not only by 1 -vector coordinates $x^{\mu}$ but also by the 2 -vector coordinates $x^{\mu \nu}, 3$-vector coordinates $x^{\mu \nu \rho} \ldots$ etc called holographic coordinates, such that

$$
\begin{aligned}
X^{M}= & s \mathbf{1}+x^{\mu} \gamma_{\mu} \\
= & x^{\mu \nu} \gamma_{\mu} \wedge \gamma_{\nu}+x^{\mu \nu \rho} \gamma_{\mu} \wedge \gamma_{\nu} \wedge \gamma_{\rho} \\
& +x^{\mu \nu \rho \sigma} \gamma_{\mu} \wedge \gamma_{\nu} \wedge \gamma_{\rho} \wedge \gamma_{\sigma},
\end{aligned}
$$

where the component $s$ is the Clifford scalar components of a polyvector valued coordinates.

Thus, in C-space proper time interval may be described as in Minkowski space [18]

$$
(d S)^{2}=(d s)^{2}+d x_{\mu} d x^{\mu}+d x_{\mu \nu} d x^{\mu \nu}+\ldots .
$$

\subsection{C-Space: Underlying Geometry}

A point in C-Space is defined as a set of holographic coordinates $\left(s, x^{\mu}, x^{\mu \nu}, \ldots\right)$ forming the coordinates of a poly-vector. Each one is expressed within bases $\left\{\gamma_{A}\right\}=\left\{1, \gamma_{a_{1}}, \gamma_{a_{1} a_{2}}, \gamma_{a_{1} a_{2} a_{3} \ldots .}\right\}, a_{1}<a_{2}<$ $a_{3}<a_{4}<a_{5}<\ldots, r=1,2,3 \ldots$, where $\gamma_{a_{1} a_{2} a_{3} \ldots}=a_{1} \wedge a_{2} \wedge a_{3} \ldots$ [23]. It is well known that the local basis $\gamma_{\mu}$, is related to the tetrad field $e_{\mu}^{a}$ such that

$$
\gamma_{\mu}=e_{\mu}^{a} \gamma_{a} .
$$

An element of a Clifford algebra is a superposition, called Clifford aggregate or poly-vector:

$$
A=a+\frac{1}{1 !} a^{\mu} \gamma_{\mu}+\frac{1}{2 !} a^{\mu \nu} \gamma_{\mu} \wedge \gamma_{\nu}+\ldots \ldots \ldots . \frac{1}{n !} a^{\mu_{1} \ldots . . \mu_{n}} \gamma_{\mu_{1}} \wedge \ldots \ldots . \gamma_{\mu_{n}} .
$$

The differential of C-space is defined as follows [21]

$$
d A=\frac{\partial A}{\partial X^{B}} d X^{B},
$$


i.e

$$
d A=\frac{\partial A}{\partial s}+\frac{\partial A}{\partial x^{\mu}} d x^{\mu}+\frac{\partial A}{\partial x^{\mu \nu}} d x^{\mu \nu}+\ldots \ldots
$$

If one takes $A=\gamma_{\mu}$, then

$$
d \gamma_{\mu}=\frac{\partial \gamma}{\partial s}+\frac{\partial \gamma_{\mu}}{\partial x^{\mu}} d x^{\mu}+\frac{\partial \gamma_{\mu}}{\partial x^{\mu \nu}} d x^{\mu \nu}+\ldots \ldots,
$$

which becomes

$$
d \gamma_{\mu}=\frac{\partial \gamma}{\partial s}+\Gamma_{\mu \nu}^{\alpha} d x^{\nu}+\Gamma_{\mu[\nu, \rho]}^{\alpha} d x^{\nu \rho}+\ldots \ldots,
$$

and can be reduced to

$$
d \gamma_{\mu}=\frac{\partial \gamma}{\partial s}+\Gamma_{\mu \nu}^{\alpha} d x^{\nu}+\frac{1}{2} R_{\beta \nu \rho}^{\alpha} d x^{\nu \rho}+\ldots \ldots,
$$

where $R_{\beta \nu \rho}^{\alpha}$ is the curvature of space-time.

Also, it can be found that for an arbitrary poly-vector $A^{M}$

$$
\frac{D A^{M}}{D x^{\mu \nu}}=\left[D_{\mu}, D_{\nu}\right] A^{M} .
$$

where $\frac{D}{D x^{\mu \nu}}$ is the covariant derivative with respect to a plane $x^{\mu \nu}$, such that

$$
\frac{D s}{D x^{\mu \nu}}=\left[D_{\mu}, D_{\nu}\right] s=K_{\mu \nu}^{\rho} \partial_{\rho} s,
$$

such that $K_{\mu \nu}^{\rho}$ is the torsion tensor.

$$
\frac{D a^{\alpha}}{D x^{\mu \nu}}=\left[D_{\mu}, D_{\nu}\right] a^{\alpha}=R_{\rho \mu \nu}^{\alpha} a^{\rho}+K_{\mu \nu}^{\rho} D_{\rho} a^{\alpha},
$$

Yet, this type of torsion (2.12) can related to the notion of torsion as mentioned by Hammond as commutator the potential associated by a prescribed scalar field $\phi[25]$

$$
K_{\mu \nu}^{\alpha}=\frac{1}{2}\left(\delta_{\mu}^{\alpha} \phi_{, \nu}-\delta_{\nu}^{\alpha} \phi_{, \mu}\right) .
$$

Thus, we can figure out that the torsion as defined in C-space (Riemannian-type) by means of the parameter(s) $s$ may act like an independent scalar field defined in the usual context of the RiemannCartan geometry.

From examining Equation (2.11) and Equation (2.12), one can find the existence of torsion tensor even in the presence of symmetric affine connection apart from its conventional notation definition of being the antisymmetric part of an affine connection as in the context of nonRiemannian geometries [13-15]. Accordingly, owing to C-space one may realize the dispute between the reliability of torsion propagating or non-propagating this can be resolved by means of of we describe torsion as a result of covariant differentiation of areas of holographic coordinates and non-propagating as being defined as anti-symmetric parts of an affine connection of poly-vectors or vectors, if one utilizes in the internal or external coordinate and its corresponding non-symmetric affine connection.

Consequently, we can regard that the geometry described within the coordinates of poly-vector described not only Riemannian but also a non-Riemannian, i.e. the composition of a Riemannian affine 
connection for the poly-vector is not necessarily Riemannian as well. This may shed some light to find out that a Riemannian Poly-vector affine connection and curvature (external coordinate capital Latin letters) may be described by non Riemannian quantities as describing its holographic coordinates (internal coordinate (Greek letters).

Accordingly, in C-space it is convenient to distinguish two frame fields [21]:

(i) Coordinate frame field, whose bases elements $\gamma_{M}, M=$ $1,2,3 \ldots .2^{n}$ depend on the position of $X$ in $\mathrm{C}$-space such that their expressions as the wedge products of vectors can not be preserved globally. Thus, one writes

$$
\gamma_{M} \equiv \gamma_{\mu_{1} \ldots \mu_{r}}
$$

The scalar product of two such basis elements gives the metric tensor of the C-space

$$
{ }^{\ddagger} \gamma_{M} \cdot \gamma_{N}=G_{M N}
$$

(ii) Orthonormal frame field, whose bases elements $\gamma_{(A)},(A)=$ $1,2,3, \ldots 2$ can at every point be expressed as a wedge product,

$$
\gamma_{(A)} \equiv \gamma_{a_{1} \ldots a_{r}}=\gamma_{a_{1}} \wedge \gamma_{a_{2}} \wedge \gamma_{a_{3} \ldots \ldots \wedge} \wedge \gamma_{a_{r}}
$$

The scalar product of the latter basis elements gives

$$
{ }^{\ddagger} \gamma_{(A)} \cdot \gamma_{(B)}=\eta_{A B}
$$

where $\eta$ is the metric tensor in flat space.

The derivative of a poly-vector is classified as follows [24]:

(i) Scalar field: Acting on a scalar field, it behaves as an ordinary partial derivative, i.e.

$$
\partial_{M} \phi=\frac{\partial \phi}{\partial X^{M}} .
$$

(ii) Coordinate bases: Acting on coordinate basis elements, it gives

$$
\partial_{M} \gamma_{N}=\Gamma_{M N}^{Q} \gamma_{Q}
$$

where $\Gamma_{M N}^{Q}$ is an affine connection. The commutator of the derivatives acting on $\gamma_{J}$ gives the Riemann tensor in $C$-space:

$$
\left[\partial_{M}, \partial_{N}\right] \gamma_{J}=R_{. J M N}^{K} \gamma_{K}
$$

(iii) Orthonormal bases (local frame field): These types of bases turn to be as follows

$$
\partial_{M} \gamma_{(A)}=-\Omega_{A}^{N} \cdot{ }_{M} \gamma_{(B)}
$$

such that $\Omega_{A}^{N}$. $M$ acts as its appropriate spin connection.

Thus, the commutator of the derivatives acting on $\gamma_{A}$ gives

$$
\left[\partial_{M}, \partial_{N}\right] \gamma_{A}=R_{. A M N}^{B} \gamma_{B}
$$

Thus, for an aggregate poly-vector $A$ one finds that:

$$
\begin{aligned}
\partial_{M}\left(A^{N} \gamma_{N}\right) & =\partial_{M} A^{N} \gamma_{N}+A^{N} \partial_{M} \gamma_{N} \\
& =\left(\partial_{M} A^{N}+\Gamma_{M K}^{N} A^{K}\right) \gamma_{N} \equiv\left(D_{M} A^{N}\right) \gamma_{N},
\end{aligned}
$$

where $D_{M} A^{N}$ is the covariant derivative acting on the plyvector components $A^{N}$. 


\subsection{C-space and the Tetrad Field}

The relationship between the two bases are related by the tetrad field $E^{A}$ associated with poly-vectors [24]

$$
\gamma_{M}=E_{M}^{(A)} \gamma_{(A)}
$$

where $E_{M}^{A}$ is the C-space vielbein, such that

$$
G_{M N}=E_{M}^{(A)} E_{N}^{(B)} \eta_{A B}
$$

Such a description does not preclude the non-Riemannian version of poly-vectors, which is a step to revisit the definition of the fundamental quantities that play an active role of describing such a type of geometry.

Accordingly, it is vital to note that the covariant derivative of Cspace associated with Vielbein of poly-vector takes the following condition [20]

$$
\partial_{N} E_{M}^{C}-\Gamma_{M N}^{P} E_{P}^{C}-E_{M}^{A} \Omega_{A}^{C} \cdot{ }_{N}=0,
$$

Consequently, the covariant derivative acting on the tetrad field is invariant under general coordinate transformation by means of $\Gamma_{N Q}^{M}$ and local Lorentz invariance as expressed in terms of $\Omega_{A}^{C}$. ${ }_{N}$ representing the spin connection in C-space.

The curvature of C-space is defined, as usually, by the commutator of derivatives acting on basis poly-vectors [23]:

$$
\left[D_{M}, D_{N}\right] \gamma_{J}=R_{M N J}^{K} \gamma_{K}
$$

or

$$
\left[D_{M}, D_{N}\right] \gamma_{(A)}=R_{M N J}^{(B)} \gamma_{(B)}
$$

where $D_{M}$ is its associate covariant derive.

Meanwhile, introducing the reciprocal basis poly-vectors $\gamma^{M}$ and $\gamma^{A}$ satisfying

$$
\begin{gathered}
\left(\gamma^{M}\right)^{\ddagger} * \gamma_{N}=\delta_{N}^{M}, \\
\left(\gamma^{(A)}\right)^{\ddagger} * \gamma_{(B)}=\delta_{(B)}^{(A)} .
\end{gathered}
$$

The components of curvature in the corresponding bases are defined as follows

$$
R_{M N J}^{K}=\partial_{M} \Gamma_{N J}^{K}-\partial_{N} \Gamma_{M J}^{K}+\Gamma_{N J}^{L} \Gamma_{M L}^{K}-\Gamma_{M J}^{L} \Gamma_{N L}^{K}
$$

or

$$
\begin{aligned}
R_{M N(A)}^{K}= & \partial_{M} \Omega_{(A) N}^{K}-\partial_{N} \Omega_{(A) M}^{K} \\
& +\Omega_{(A) N}^{(C)} \Omega_{(C) M}^{K}-\Omega_{(A) M}^{(C)} \Omega_{(C) N}^{K}
\end{aligned}
$$

The latter expression is a generalization to $C$-space of the analogous expression in Riemannian geometry for manifolds of point-like objects $[26]$.

Moreover, there is a counterpart version of non-vanishing curvature and torsion as defined in non-Riemannian geometry, in C-space leading to define torsion of poly-vectors to become

$$
\Lambda_{M N}^{J}=\bar{\Gamma}_{M N}^{J}-\bar{\Gamma}_{N M}^{J}
$$


Also, the contortion of poly-vectors $\Omega_{B C M}$ is given by

$$
\Omega_{B C M}=\frac{1}{2} E_{M}^{(A)}\left(\Delta_{[(A)(B)](C)}-\Delta_{[(B)(C)](A)}+\Delta_{[(C)(A)](B)}\right)
$$

where $\Delta_{[(A)(B)](C)}$ is defined as the Ricci coefficient of rotation [23]

$$
\Delta_{[(A)(B)](C)}=E_{(A)}^{M} E_{(B)}^{N}\left(\partial_{M} E_{N(C)}-\partial_{M} E_{N(C)}\right) .
$$

Moreover, another covariant derivative associated with nonsymmetric affine connection $\bar{\Gamma}_{N K}^{M}$ is defined as follows

$$
X_{\mid N}^{M}=\partial_{N} X^{M}+\bar{\Gamma}_{N S}^{M} X^{S},
$$

such that

$$
\begin{gathered}
\bar{\Gamma}_{N S}^{M} X^{S}=\Gamma_{N S}^{M} X^{S}+\Delta_{N S}^{M} \\
X_{\mid N S}^{M}-X_{\mid S N}^{M}=\bar{R}_{Q N S}^{M} X^{Q}+\Lambda_{N S}^{Q} X_{\mid Q}^{M} .
\end{gathered}
$$

such that

$$
R_{M N J}^{K}=\partial_{M} \bar{\Gamma}_{N J}^{K}-\partial_{N} \bar{\Gamma}_{M J}^{K}+\bar{\Gamma}_{N J}^{L} \bar{\Gamma}_{M L}^{K}-\bar{\Gamma}_{M J}^{L} \bar{\Gamma}_{N L}^{K} .
$$

Due to richness of these quantities this type work will be going to examine the behavior of extended objects subjects having sensitivity to these quantities in our future work ; while in our present work we focus on deriving the equations of motion and their deviation paths for different extended objects spinning and charged for poly-vectors defined within the context of Riemannian-like C-Space as explained in the following sections .

\subsection{C-Space: An Arena of Unifying Physics}

It is worth mentioning that, physical objects considered as matter in space-time, can be in the form of membranes (brane) of various dimensions (p-branes) [18]

From this perspective, the notation of Clifford aggregate as described in (2.1) , it may be important to revisit the conventional notations of mass and charge in the presence of $\mathrm{C}$-space to be

$$
M=m \mathbf{1}+p^{\mu} \gamma_{\mu}+S^{\mu \nu} \gamma_{\mu \nu},
$$

and

$$
\epsilon=e \mathbf{1}+A^{\mu} \gamma_{\mu}+F^{\mu \nu} \gamma_{\mu \nu},
$$

where $M$ and $\epsilon$ are mass and charge of extended objects respectively.

Nevertheless, it is vital to be noted that in Clifford Space, there is a striking virtue, unlike the conventional string theory which expressed within 26 dimensions in which gauge fields are described as compact dimensions; while in C-space there is only 16 non-compact dimensions. Consequently, Pavsic (2005) generalized the conventional 4-dimensional space-time into 16 dimensional à la Klauza-Klein theory [23].

(i) There is no need for extra-dimensions of its corresponding spacetime.

(ii) There is no need to have compact extra-dimensions. The extradimensions of C-space, namely $x^{\mu}, x^{\mu \nu}, x^{\mu \nu \rho}$, and $x^{\mu \nu \rho \sigma}$ describe the extended objects, therefore they are regarded as physical dimensions. 
(iii) The number of components $G_{\mu \tilde{M}}, \mu=0,1,2,3$ and $\tilde{M} \neq \mu$ is 12 , which is the same as the number of gauge fields in the standard models.

Thus, the line element of poly-vectors in C-space become

$$
|d X|^{2}=d S^{2}=G_{M N} d X^{M} d X^{N},
$$

i.e.

$$
d S^{2}=d s^{2}+L^{-2} d x_{\mu} d x^{\mu}+L^{-4} d x_{\mu \nu} x^{\mu \nu}+L^{-6} d x_{\mu \nu \rho} x^{\mu \nu \rho}+\ldots
$$

where $G_{M N}=E_{M}^{\dagger} E_{N}$ is C-space metric, $L$ is the Planck length [12]. Consequently, Equation (2.43) can be expressed as

$$
\begin{aligned}
d S^{2}= & c^{2} d t^{2}\left(1+\frac{L^{-2}}{c^{2}} \frac{d x_{\mu}}{d t} \frac{d x^{\mu}}{d t}\right. \\
& \left.+\frac{L^{-4}}{c^{2}} \frac{d x_{\mu \nu}}{d t} \frac{d x^{\mu \nu}}{d t}+\frac{L^{-6}}{c^{2}} \frac{d x_{\mu \nu \rho}}{d t} \frac{d x^{\mu \nu \rho}}{d t}+\ldots\right)
\end{aligned}
$$

with taking into account that the parameter $s$ is described only in the ordinary space-time i.e. $(d s)^{2}=g_{\mu \nu} d x^{\mu} d x^{\nu}[22]$.

Meanwhile, in C-space, Castro and Pavsic (2005), showed that, there are some principles must be revisited especially the speed of light which is no longer the upper limit to be reached, but another combination of constants made of Planck's length in the following way [18]:

(i) Maximum 1-vector speed $\frac{d x^{\mu}}{d s}=3 \times 10^{8} \mathrm{~ms}^{-1}$,

(ii) Maximum 2-vector speed $\frac{d x^{\mu \nu}}{d s}=1.6 \times 10^{-35} \mathrm{~m}^{2} \mathrm{~s}^{-1}$,

(iii) Maximum 3-vector speed $\frac{d x^{\mu \nu \rho}}{d s}=7.7 \times 10^{-62} \mathrm{~m}^{3} \mathrm{~s}^{-1}$,

(iv) Maximum 4-vector speed $\frac{d x^{\mu \nu \rho}}{d s}=7.7 \times 10^{-96} \mathrm{~m}^{4} \mathrm{~s}^{-1} \ldots$ etc.

Also, it is worth mentioning that by means of C-space a particle as observed from 4-dimensional space, that can be speed of particles may be more than the conventional speed of light due to involvement of holographic coordinates $x^{\mu \nu}, x^{\mu \nu \rho} \ldots$ etc ; but does not exceeds the modified speed of light due to C-space. This can lead us to consider there will be specific upper limit to objects due to p-brans as $p=$ $0,1,2,3, \ldots$

The Line element of poly-vectors in C-space is defined as follows [23]:

$$
d S^{2}=G_{M N} d X^{M} \times d X^{N}
$$

provided that the matrix $G_{M N}$ is defined as

$$
G_{M N}=\left[\begin{array}{cc}
g_{\mu \nu} & G_{\mu \bar{N}} \\
G_{\bar{M} \nu} & G_{\bar{M} \bar{N}}
\end{array}\right] .
$$

These degrees of freedom are in principle not hidden by which we describe the extended objects, therefore we do not need to compact dimensions of its internal space.

The metric of C-space $G_{M N}$ is subdivided into $G_{\mu \nu}=g_{\mu \nu}$ which relates to gravity, while gauge fields $G_{\mu \bar{M}}$, where $\mu \neq \bar{M}$ assumes 12 possible values, excluding the four values of $\nu=0,1,2,3$, and other 12 gauge fields ,to be defined as follows: 1 photon, 3 weak gauge bosons and 8 gluons described $A_{\mu}, A_{\mu}^{a}, a=1,2,3$ and by $A_{\mu}^{c} c=1,1,3, \ldots 8$. respectively.

It can be found that the number of mixed components in $G_{\mu \bar{M}}=$ $\left(G_{\mu 1}, G_{\mu[\alpha \beta]}, G_{\mu[\alpha \beta \gamma]}, G_{\mu[\alpha \beta \gamma \delta]}\right)$ of Clifford metric coincides with the 
number of gauge fields in the standard model. For a fixed $\mu$, there are 12 mixed components of $G_{\mu \bar{M}}$ and 12 gauge fields $A_{\mu}^{a}, W_{\mu}^{c}, A_{\mu}^{c}$. This coincidence is fascinating and it may indicate that the known interactions are incorporated in curved Clifford space. The number of mixed metric $G_{\mu \bar{M}}$ is 12 the same numbers as the number of gauge fields in the standard model.

In addition, there are also interaction due to components $G_{\bar{M}} \bar{N}$ but do not have the properties of Yang-Mills fields. Thus, Pavsic (2006) has considered it as a metric of an internal space [24], which may be a glimpse to express bi-metric theory of gravity within the context of C-space. Such a study will be examined in our future work.

\section{Trajectories of Poly-Vectors in C-Space}

\subsection{Equations of Motion in C-Space}

Pavsic [24] considered the classical action for a point particle in Cspace:

$$
I\left[X^{\mu}, G_{M N}\right]=\int d \tau \sqrt{\left.\left[G_{M N} \dot{X}^{M} \dot{X}^{N}\right)\right]}+\frac{\kappa}{16 \pi} \int d[x] R .
$$

The above classical action is a combined action for path equations and gravitational field equations respectively. One obtains its path equation by taking the variation with respect to $X^{C}$ to der ve corresponding geodesic equation i.e.

$$
\frac{1}{\sqrt{\dot{X}^{2}}} \frac{d}{d \tau} \frac{\dot{X}^{M}}{\sqrt{\dot{X}^{2}}}+\Gamma_{N K}^{M} \frac{\dot{X}^{M} \dot{X}^{N}}{\dot{X}^{2}}=0 .
$$

Also, from the same function (3.47) by taking the variation with respect to $G_{M N}$ one obtains its corresponding field equation

$$
R^{M N}-\frac{1}{2} G^{M N} R=8 \pi \kappa \int \delta_{(C)}(x-X) \dot{X}^{M} \dot{X}^{N},
$$

where $\delta_{(C)}$ is the delta function in $\mathrm{C}$-space. The latter equation can be expressed as

$$
R^{M N}-\frac{1}{2} G^{M N} R=8 \pi T_{M N},
$$

where $T_{M N}$ is the energy-momentum tensor as defined in C-space.

\subsection{C-Space and Problem of Motion: Pezzaglia's Approach}

As an introductory step to the C-space formalism obtained by CastroPavsic [18], it is worth to mention that Pezzaglia had presented a speculative vision about the need to utilize Clifford space, the problem of unification can be passed by stages of composing scalar, vectors and bi-vectors in one form with taking into consideration that the corresponding bases vectors are described in non-orthonormal curved space in the following way [11].

$$
\gamma_{\mu} \cdot \gamma_{\nu} \equiv \frac{1}{2}\left\{\gamma_{\mu} \gamma_{\nu}\right\}=\frac{1}{2}\left\{\gamma_{\mu} \gamma_{\nu}+\gamma_{\nu} \gamma_{\mu}\right\}=g_{\mu \nu}
$$

where $g_{\mu \nu}$ is the metric tensor which may be a function of position. The outer product of two different bases vectors yields a new object 
called a bi-vector, which is the basis of a plane spanned by two basis vectors

$$
\gamma_{\mu \nu}=-\gamma_{\nu \mu}=\equiv \gamma_{\mu} \wedge \gamma_{\nu} \equiv \frac{1}{2}\left[\gamma_{\mu} \gamma_{\nu}\right]=\frac{1}{2}\left(\gamma_{\mu} \gamma_{\nu}-\gamma_{\nu} \gamma_{\mu}\right)=s_{\mu \nu},
$$

where $s_{\mu \nu}$ acts as the skew symmetric part of the metric $g_{\mu \nu}$ i.e.

$$
g_{[\mu \nu]}=s_{\mu \nu} .
$$

This illustration can be clarified by describing the momentum polyvector and spin bi-vector angular momentum

$$
M \equiv \frac{1}{\lambda} p^{\mu} \gamma_{\mu}+\frac{1}{\lambda^{2}} S^{\mu \nu} \gamma_{\mu \nu}
$$

such that $\lambda$ is a universal length constant

$$
p^{\mu}=m \frac{d x^{\mu}}{d s},
$$

and

$$
S^{\mu \nu}=m \frac{d a^{\mu \nu}}{d s},
$$

where $S^{\mu \nu}$, in which is defined to be a bi-vector coordinate. In flat space one finds its corresponding equation of motion

$$
\dot{M}^{\mu}=\frac{d M^{\mu}}{d s}=0,
$$

i.e.

$$
\dot{p}^{\mu}=\frac{d p^{\mu}}{d s}=0,
$$

and

$$
\dot{S}^{\mu \nu}=\frac{d S^{\mu \nu}}{d s}=0 .
$$

It is worth mentioning that the modified momentum which include both linear and spin angular momentum may give rise to describe the momentum of the extended object not consider it as a test particle but a dipole one has an impact on its spinning case. Thus, studying momentum with taking its spinning motion may give rise to regard the spinning of the particle increases its mass as shown in Equation (3.51) and Equation (3.54) [12]. Now, the arising question is the situation of these equations in the presence of gravitational field, which may be expressed as follows

$$
\frac{d^{2} x^{\mu}}{d s^{2}}+\Gamma_{\nu \rho}^{\mu} \frac{d x^{\nu}}{d s} \frac{d x^{\rho}}{d s}+\Gamma_{\nu[\rho, \delta]}^{\mu} \frac{d x^{\nu}}{d s} \frac{d a^{\rho \delta}}{d s}=0
$$

to become in the following way

$$
\frac{d^{2} x^{\mu}}{d s^{2}}+\Gamma_{\nu \rho}^{\mu} \frac{d x^{\nu}}{d s} \frac{d x^{\rho}}{d s}+\frac{1}{2 m} R_{\nu \rho \delta}^{\mu} \frac{d x^{\nu}}{d s} S^{\rho \delta}=0
$$

and

$$
\begin{gathered}
\frac{d S^{\mu \nu}}{d s}+\Gamma_{\sigma \rho}^{\mu} S^{\sigma \nu} \frac{d x^{\rho}}{d s}+\Gamma_{\sigma \rho}^{\nu} S^{\mu \sigma} \frac{d x^{\rho}}{d s}=0, \\
\frac{D^{2} x^{\mu}}{D s^{2}}=\frac{1}{2 m} R_{\nu \rho \sigma}^{\mu} S^{\rho \sigma} \frac{d x^{\nu}}{d s},
\end{gathered}
$$

and

$$
\frac{D S^{\mu \nu}}{D s}=\frac{d a^{\alpha \beta}}{d s}\left(\Gamma_{\alpha[\beta, \sigma]}^{\mu} S^{\sigma \nu}+\Gamma_{\alpha[\beta, \sigma]}^{\nu} S^{\mu \sigma}\right)=0,
$$


i.e.

$$
\frac{D S^{\mu \nu}}{D s}=\frac{1}{2 m} S^{\alpha \beta}\left(R_{\alpha \beta \sigma}^{\mu} S^{\sigma \nu}+R_{\alpha \beta \sigma}^{\nu} S^{\mu \sigma}\right),
$$

such that $\frac{D}{D s}$ is the conventional covariant derivative as defined in Riemannian Space. It can be figured out that the Equation (3.60) acts exactly as the Papapetrou equation for short; while extra terms in terms of curvature and spin tensor are added in Equation (3.61).

Following this stream of thinking in case of electro-magnetic field in the presence of flat space, one my find out that

$$
\frac{d^{2} x^{\mu}}{d s^{2}}=\frac{e}{m} F_{\nu}^{\mu} \frac{d x^{\nu}}{d s}
$$

and

$$
\dot{S}^{\mu \nu}=\frac{d S^{\mu \nu}}{d s}=\frac{e}{m} F_{\rho}^{[\mu} S^{\nu] \rho},
$$

where $F_{\mu \nu}$ is the elecro mangnetic tensor, which turns to be in case of gravitational field as

$$
\frac{D^{2} x^{\mu}}{D s^{2}}=\frac{1}{2 m} R_{\nu \rho \delta}^{\mu} S^{\rho \delta} \frac{d x^{\nu}}{d s}+\frac{e}{m} F^{\mu \nu} \frac{d x^{\nu}}{d s},
$$

and

$$
\frac{D S^{\mu \nu}}{D s}=\frac{e}{m} F_{\rho}^{[\mu} S^{\nu] \rho}+\frac{1}{2} \frac{d a^{\alpha \beta}}{d s}\left(R_{\alpha \beta \sigma}^{\mu} S^{\sigma \nu}+R_{\alpha \beta \sigma}^{\nu} S^{\mu \sigma}\right),
$$

such that

$$
\frac{D S^{\mu \nu}}{D s}=\frac{e}{m} F_{\rho}^{[\mu} S^{\nu] \rho}+\frac{1}{2 m} S^{\alpha \beta}\left(R_{\alpha \beta \sigma}^{\mu} S^{\sigma \nu}+R_{\alpha \beta \sigma}^{\nu} S^{\mu \sigma}\right) .
$$

Thus, it can be shown that Equation (3.66) acts as the Dixon equation, extra terms in terms of curvature and spin tensor are added in Equation (3.67). From studying these equations, we can see the involvement of bi-vector to be affected by additional factors of curvature of the space-time and spin tensor. This may give rise to make sure that in case of tri-vectors and other multi-vectors these effects will be added. Such an argument may impose the necessity to find a general formula capable to embody all these effects. It was found that using poly-vector coordinates may represent all these forms in a compact way. So it is essential to display the underlying geometry of polyvectors prior to examining the corresponding equations of motion and the deviation ones.

\subsection{The Bazanski Approach for Poly-Vectors}

Equations of geodesic and geodesic deviation equations in Riemannian geometry are required to examine many problems of motion for different test particles in gravitational fields. This led many authors to derive them by various methods, one of the most applicable ones is the Bazanski approach [27] in which from one single Lagrangian one can obtain simultaneously equation of geodesic and geodesic deviations which has been applied in different theories of gravity [4-9], and [28-30]. Thus, by analogy this technique in case of Poly-vectors to become [31],

$$
L=G_{M N} U^{M} \frac{D \Psi^{N}}{D S},
$$

where, $G_{M N}$ is the metric tensor, $U^{M}=\frac{d X^{M}}{d S}$, is a unit tangent polyvector of the path whose parameter is $S$, and $\Psi^{\nu}$ is the deviation 
poly-vector associated to the path $(S), \frac{D}{D S}$ is the covariant derivative with respect to parameter $S$.

Applying the Euler Lagrange equation, by taking the variation with respect to the deviation poly-vector $\Psi^{C}$,

$$
\frac{d}{d S} \frac{\partial L}{\partial \dot{\Psi}^{C}}-\frac{\partial L}{\partial \Psi^{C}}=0
$$

to obtain the geodesic equation

$$
\frac{D U^{C}}{D S}=0
$$

and taking the variation with respect the the unit poly-vector $U^{C}$,

$$
\frac{d}{d S} \frac{\partial L}{\partial U^{C}}-\frac{\partial L}{\partial x^{C}}=0
$$

to obtain the geodesic deviation equation,

$$
\frac{D^{2} \Psi^{E}}{D S^{2}}=R_{A B C}^{E} U^{A} U^{B} \Psi^{C},
$$

where $R_{B C D}^{A}$ is Riemann-Christoffel tensor described by poly-vectors.

\subsection{Geodesic and Geodesic Deviation of C-Space: An Alternative Approach}

Equations geodesic deviation of poly-vector are derived in a way of modifying the conventional Bazanski method, using the commutation relations and a condition between deviation poly-vector and polyvector as well. This method has been worked successfully to derive deviation equation for extended objects with wobbling as shown in [6-9].

Applying the usual commutation relation [32] on Equation (3.70) we obtain:

$$
\frac{D}{D \Omega} \frac{D A^{D}}{D S}-\frac{D}{D S} \frac{D A^{D}}{D \Omega}=\bar{R}_{B C E}^{D} A^{B} U^{C} \Psi^{E}
$$

provided that

$$
\frac{D U^{A}}{D S}=\frac{D \Psi^{A}}{D \Omega} .
$$

Thus, we obtain its corresponding geodesic deviation equations

$$
\frac{D^{2} \Psi^{C}}{D S^{2}}=R_{B D E}^{C} U^{B} U^{D} \Psi^{E} .
$$

\section{Transformation of Geodesic Poly-Vectors to Charged and Spinning Poly-Vectors}

\subsection{From Geodesic Poly-Vector to Charged Object Poly-Vector}

In this approach we are going to utilize the famous application for transforming geodesics to spinning object differently [32] to become reliable for charged object. In this case, one can assume that charged object travels on a deviated trajectory belongs to family of deviation parameters such that

$$
W^{C}=U^{C}+\frac{\epsilon}{M} \frac{D \Psi^{C}}{D S}
$$


where $W^{C}=\frac{d X^{C}}{d \Xi}$.

Thus, differentiating both sides by $\frac{D}{D \bar{S}}$ and taking that $\frac{d S}{d \Xi}=1$, one obtains,

$$
\frac{D W^{C}}{D \bar{\Xi}}=\frac{D}{D S}\left[U^{C}+\frac{\epsilon}{M} \frac{D \Psi^{C}}{D S}\right] \frac{d S}{d \bar{\Xi}} .
$$

Now, if we consider electro-magnetic tensor as follows

$$
F^{A B} U^{B}=\left(R_{B D E}^{A} U^{D} \Psi^{E}\right) U^{B},
$$

then Equation (4.75) becomes

$$
\frac{D W^{C}}{D \sigma}=\frac{\epsilon}{M} F_{. B}^{C} U^{B} \frac{d S}{d \bar{\Xi}}
$$

which becomes the equivalent Lorentz force (Charged object) in Curved Clifford Space

$$
\frac{D W^{C}}{D \Xi}=\frac{\epsilon}{M} F^{A B} W^{B}
$$

\subsection{From Geodesic Poly-Vector to Spinning Poly-Vectors}

If we consider spinning poly-vectors are regarded as geodesics transferred from one path defined by a parameter $S$ into another one $\bar{S}$, such a translation may cause the object behave as a spinning particle rather moving as a test particle. We suggest the following poly-vector $V^{C}$ defined in the following way

$$
V^{C}=U^{C}+\beta \frac{D \Psi^{C}}{D S} .
$$

By taking the covariant derivative on both sides we obtain, with taking into account $\frac{d S}{d \bar{S}}=1$,

$$
\frac{D V^{C}}{D \bar{S}}=\frac{D}{D S}\left[U^{C}+\beta \frac{D \Psi^{C}}{D S}\right] \frac{d S}{d \bar{S}} .
$$

Assuming that $\beta=\frac{\bar{S}}{M}$, and $\tilde{S}$ is the magnitude of the spin tensor as defined by

$$
S^{A B}=\tilde{S}\left[U^{A} \Psi^{B}-U^{B} \Psi^{A}\right] .
$$

Accordingly, substituting Equations (3.70), (3.72) and (4.83) into (4.82), provided that $\frac{d S}{d \bar{S}}=1$, we get after some manipulations:

$$
\frac{D U^{C}}{D S}=\frac{1}{2 m} R_{B D E}^{C} U^{B} S^{D E},
$$

which is the corresponding Papapetrou equation, for short, in C-space.

\section{The Bazanki Approach for Extended Objects of C-Space}

\subsection{Equations of Charged and Charged Deviation Equations in C-Space}

The Bazanski approach to obtain an equation of a charged object may be found as follows:

$$
L=G_{A B} W^{A} \frac{D \Phi^{B}}{D \Xi}+\frac{\epsilon}{M} F_{A B} W^{A} \Phi^{B} .
$$


Taking the variation with respect to $\Phi^{C}$ and $W^{C}$ we obtain after some manipulations one finds

$$
\frac{D W^{C}}{D S}=F^{C B} W^{B}
$$

and

$$
\frac{D^{2} \Phi^{C}}{D \bar{S}^{2}}=R_{B D E}^{C} W^{E} W^{D} \Phi^{E}+\frac{\epsilon}{M}\left(F^{C B} W^{B}\right)_{; D} \Phi^{D} .
$$

\subsection{Spinning and Spinning Deviation Equations of C-Space}

We suggest the equivalent Bazanski Lagrangian for deriving the equations for spinning and spinning poly-vectors to be

$$
L=G_{A B} P^{A} \frac{D \Psi^{B}}{D \bar{S}}+S_{A B} \frac{D \Psi^{A B}}{D \bar{S}}+F_{A} \Psi^{A}+M_{A B} \Psi^{A B},
$$

such that

$$
P^{A}=m U^{A}+U_{\beta} \frac{D S^{A B}}{D S},
$$

where $P^{\mu}$ is the momentum poly-vector $F^{\mu}=\frac{1}{2} R_{\nu \rho \delta}^{\mu} S^{\rho \delta} U^{\nu}, R_{\beta \rho \sigma}^{\alpha}$ is the Riemann curvature, $\frac{D}{D S}$ is the covariant derivative with respect to a parameter $\bar{S}, S^{\alpha \beta}$ is the spin poly-tensor, and $M^{\mu \nu}=P^{\mu} U^{\nu}-P^{\nu} U^{\mu}$ such that $U^{\alpha}=\frac{d x^{\alpha}}{d s}$ is the unit tangent poly-vector to the geodesic one. In a similar way as performed in (5.83), by taking the variation with respect to $\Psi^{\mu}$ and $\Psi^{\mu \nu}$ simultaneously in (5.86) one obtains

$$
\frac{D P^{M}}{D \bar{S}}=F^{M}
$$

and

$$
\frac{D S^{M N}}{D \bar{S}}=M^{M N}
$$

Using the following identity

$$
A_{; N H}^{D}-A_{; H N}^{D}=R_{B N H}^{D} A^{B},
$$

on both Equation (5.87) and Equation (5.88), such that $A^{D}$ is an arbitrary poly-vector. Also, multiplying both sides with arbitrary polyvectors, $U^{H}$ and $\Psi^{B}$ as well as using the following condition [33]

$$
U_{; H}^{A} \Psi^{H}=\Psi_{; H}^{A} U^{H}
$$

and $\Psi^{A}$ is its deviation poly-vector associated to the unit poly-vector tangent $U^{A}$. And in a similar we can find out that:

$$
S_{; H}^{A B} \Psi^{H}=\Psi_{; H}^{A B} U^{H} .
$$

Consequently, one obtains the corresponding deviation equations which are inspired from the workings of Mohseni [34]

$$
\frac{D^{2} \Psi^{A}}{D S^{2}}=\bar{R}_{B H C}^{A} P^{B} U^{H} \Phi^{C}+F_{; H}^{A} \Psi^{H},
$$

and

$$
\frac{D^{2} \Psi^{A B}}{D \bar{S}^{2}}=S^{[B D} R_{D H C}^{A]} U^{H} \Psi^{C}+M_{; H}^{A B} \Psi^{H}
$$




\subsection{The Generalized Dixon Equation in C-Space}

In this part, we suggest the following Lagrangian which enables us to obtain the spinning charged poly-vector in Clifford space.

$$
L=G_{A B} P^{A} \frac{D \Psi^{B}}{D S}+S_{A B} \frac{D \Psi^{A B}}{D S}+\tilde{F}_{A B} \Psi^{A} U^{B}+\tilde{M}_{A B} \Psi^{A B},
$$

such that

$$
\tilde{F}_{A B}=\frac{1}{m}\left(e F_{A B}+\frac{1}{2} R_{A B C D} S^{C D}\right)
$$

and

$$
\tilde{M}_{A B}=\left(P_{A} U^{B}-P_{B} U^{U}+F_{A C} S_{. B}^{C}-F_{A C} S_{A}^{\cdot}{ }^{C}\right) .
$$

Taking the variation with respect to $\Psi^{\mu}$ and $\Psi^{\mu \nu}$ simultaneously in (5.94) we obtain

$$
\begin{gathered}
\frac{D P^{M}}{D S}=\frac{\epsilon}{M} F_{B}^{M} U^{B}+\frac{1}{2 M} R_{N E Q}^{M} S^{E Q} U^{N}, \\
\frac{D S^{M N}}{D S}=P^{M} U^{N}-P^{N} U^{M}+F^{M C} S_{C}{ }^{N}-F^{C N} S_{.}^{M}{ }_{C} .
\end{gathered}
$$

Thus, applying the same laws of commutation as illustrated in (5.92) and (5.93) we obtain their corresponding deviation equations

$$
\begin{aligned}
\frac{D^{2} \Psi^{A}}{D S^{2}}= & R_{B H C}^{A} P^{B} U^{H} \Phi^{C} \\
& +\left(\frac{\epsilon}{M} F_{B}^{M} U^{B}+\frac{1}{2 m} R_{N E Q}^{M} S^{E Q} U^{N}\right)_{; H} \Psi^{H},
\end{aligned}
$$

and

$$
\begin{aligned}
\frac{D^{2} \Psi^{A B}}{D S^{2}}= & S^{[B D} R_{D H C}^{A]} U^{H} \Psi^{C}+\left(P^{M} U^{N}-P^{N} U^{M}\right. \\
& +F^{M C} S_{C}{ }^{N}-F^{C N} S_{\left.\cdot{ }_{C}\right)_{; H} \Psi^{H}} .
\end{aligned}
$$

\section{Concluding Remarks}

In our approach, we have obtained the relevant equations of spinning and spinning of charged poly-vectors, as well as their deviation equations in C-space i.e. (4.84), (5.97) and (5.98). This type of work is regarded an extension to a previous work, obtaining equations of spinning and spinning objects in Riemannian and non-Riemannian geometries, using the Bazanski approach [4-9].

Throughout, this study, it has been found the necessity to regard extended objects, the most reliable ones to express the actual nature of objects, rather than relying to a point-like system. Due to this transition, the associate building block of space-time has to be revisited in the context of $\mathrm{C}$-space. This approach has served to redefine different notations and quantities like mass and charge of any object as shown in (2.41) and (2.42). Not only the definitions of mass and charge for any object are needed to be revised, but also the notation of the torsion of space-time as defined in the internal coordinates (2.13) is obtained due to imposing the rules of differentiation of Clifford space as shown in equations $(2.10),(2.11)$ and (2.12); while their corresponding external coordinates (poly-vector) are purely Riemannaian. This is due to taking the covariant derivative affected oriented areas $x^{\mu \nu}$ of holographic coordinates as shown in (2.10), and (2.11) from (2.12). Consequently, 
the meaning of torsion is becoming as similar as being obtained in terms of commutation relation of differential operator on a potential scalar field as expressed by Hammond [25] on dealing with internal coordinates. While, the conventional definition of torsion (2.35) in the context of non-symmetric affine connection is also preserved, if one applies the associate geometry describing the external coordinates as a non-Riemaniann geometry [13-17].

Moreover, we have extended our study to derive the equivalent charged spinning equations and their deviation ones for poly-vectors in C-space, to be considered its corresponding generalized Dixon equation (5.97) and (5.98) in which their corresponding deviations (5.99) and (5.100) are obtained. This approach may help to examine the stability of extended objects in C-space.

To sum up, the problem of motion in C-space becomes a paradigm shift towards identifying the behavior of objects in a deterministic way which can be detected in terms of both internal coordinates and their external ones. Such a tendency may inspire many scientists to find out such a unified theory describing both micro-physics and macro-physics in one form.

\section{Conflicts of Interest}

The author declares no conflicts of interest regarding the publication of this paper.

\section{References}

[1] Mathisson, M. (1937) Acta Physica Polonica, 6, 163-209. (In German)

[2] Papapetrou, A. (1951) Proceedings of the Royal Society of London. Series A, 209, 248. https://doi.org/10.1098/rspa.1951.0200

[3] Dixon, W.G. (1970) Proceedings of the Royal Society of London. Series A, 314, 499. https://doi.org/10.1098/rspa.1970.0020

[4] Kahil, M.E. (2006) Journal of Mathematical Physics, 47, Article ID: 052501. https://doi.org/10.1063/1.2196749

[5] Pavšič, M. and Kahil, M.E. (2012) Open Physics, 10, 414.

[6] Kahil, M.E. (2018) Gravitation and Cosmology, 24, 83.

[7] Kahil, M.E. (2018) ADAP, 3, 136.

[8] Kahil, M.E. (2020) Gravitation and Cosmology, 26, 241-248. https://doi.org/10.1134/S0202289320030093

[9] Kahil, M.E. (2020) Indian Journal of Physics. https://doi.org/10.1007/s12648-020-01793-5

[10] Kahil, M.E. (2015) Odessa Astronomical Publications, 28, 126. https://doi.org/10.18524/1810-4215.2015.28.70600

[11] Pezzaglia, W. (1997) Physical Applications of a Generalized Clifford Calculus (Papapetrou Equations and Metamorphic Curvature). arXiv:gr-qc/9710027

[12] Pezzaglia, W. (1999) Dimensionally Democratic Calculus and Principles of Polydimensional Physics. arXiv:gr-qc/99120251. 
[13] Mikhail, F.I. and Wanas, M.I. (1977) Proceedings of the Royal Society of London. Series A, 356, 471.

[14] Wanas, M.I. (2000) Studii Si Cercetari Stiintifice. Seria Matematica, No. 10, 297-309.

[15] Hayashi, K. and Shirifuji, T. (1979) Physical Review D, 19, 3524. https://doi.org/10.1103/PhysRevD.19.3524

[16] Hehl, F.W., von der Heyde, P., Kerlik, G.D. and Nester, J.M. (1976) Reviews of Modern Physics, 48, Article ID: 393416.

[17] Hehl, F.W. (1980) Four Lectures on Poincare Gauge Field Theory. In: Bergmann, P.G. and De Sabbata, V., Eds., Cosmology and Gravitation. NATO Advanced Study Institutes Series (Series B. Physics), Vol. 58, Springer, Boston, MA, 5-61. https://doi.org/10.1007/978-1-4613-3123-0_2

[18] Castro, C. and Pavšič, M. (2005) Progress in Physics, 1, 32.

[19] Castro, C. (2012) Advances in Applied Clifford Algebras, 23, 3962. https://doi.org/10.1007/s00006-012-0370-4

[20] Castro, C. (2006) Annals of Physics, 321, 813-839.

https://doi.org/10.1016/j.aop.2005.11.008

[21] Castro, C. and Pavšič, M. (2002) Physics Letters B, 539, 133-142. https://doi.org/10.1016/S0370-2693(02)02068-3

[22] Castro, C. (2017) Advances in Applied Clifford Algebras, 27, 2393-2405. https://doi.org/10.1007/s00006-017-0763-5

[23] Pavšič, M. (2005) Physics Letters B, 614, 85-95. https://doi.org/10.1016/j.physletb.2005.03.052

[24] Pavšič, M. (2007) Journal of Physics: Conference Series, 66, Article ID: 012022.

https://doi.org/10.1088/1742-6596/66/1/012022

[25] Hammond, R. (2002) Reports on Progress in Physics, 65, 599. https://doi.org/10.1088/0034-4885/65/5/201

[26] Collins, P.D., Martin, A.D. and Squires, E.J. (1989) Particle Physics and Cosmology. John Wily and Sons Inc., Hoboken.

[27] Bazanski, S.L. (1989) Journal of Mathematical Physics, 30, 1018. https://doi.org/10.1063/1.528370

[28] Wanas, M.I., Melek, M. and Kahil, M.E. (1995) Astrophysics and Space Science, 228, 273-276. https://doi.org/10.1007/BF00984980

[29] Wanas, M.I., Melek, M. and Kahil, M.E. (2000) Gravitation and Cosmology, 6, 319 .

[30] Kahil, M.E. (2017) Gravitation and Cosmology, 23, 70-79. https://doi.org/10.1134/S0202289317010066

[31] Kahil, M.E. (2007) AIP Conference Proceedings, 957, 329-332.

[32] Bini, D. and Geralico, A. (2011) Physical Review D, 84, Article ID: 104012. https://doi.org/10.1103/PhysRevD.84.104012 
[33] Heydrai-Fard, M., Mohseni, M. and Sepanigi, H.R. (2005) Physics Letters B, 626, 230-234.

https://doi.org/10.1016/j.physletb.2005.08.098

[34] Mohseni, M. (2010) General Relativity and Gravitation, 42, 24772490. https://doi.org/10.1007/s10714-010-0995-3 